Service social

\title{
Appauvrissement, aide alimentaire et organismes communautaires. De la compréhension à l'action
}

\section{Élaine Côté, Sonia Racine et Joël Rouffignat}

Volume 44, numéro 3, 1995

Pauvreté

URI : https://id.erudit.org/iderudit/706708ar

DOI : https://doi.org/10.7202/706708ar

Aller au sommaire du numéro

\section{Éditeur(s)}

École de service social de l'Université Laval

ISSN

1708-1734 (numérique)

Découvrir la revue

Citer cet article

Côté, É., Racine, S. \& Rouffignat, J. (1995). Appauvrissement, aide alimentaire et organismes communautaires. De la compréhension à l'action. Service social, 44(3), 95-114. https://doi.org/10.7202/706708ar

\section{Résumé de l'article}

L'aide alimentaire connaît une croissance sans précédent dans les sociétés dites développées. C'est également le cas au Québec où les activités des banques alimentaires ont connu une croissance quasi exponentielle pendant la dernière décennie. Le réseau d'organismes communautaires qui effectuent la distribution de cette aide alimentaire doit s'interroger et être critique quant à la place et à la finalité de cette aide pour l'atteinte de ses objectifs, dans son fonctionnement quotidien et dans ses pratiques sociales de dépannage. L'appauvrissement des ménages est bien sûr une réalité incontournable, tout comme le développement d'un nouveau bénévolat riche en solidarité sociale est significatif, mais ces actions d'aide sont aisément récupérables financièrement et idéologiquement par un État qui retraite sur ses politiques sociales. Une recherche conscientisante ayant à son origine la Table d'interaction sur la faim de Québec et Moisson Québec a été l'un des moyens utilisés pour que les intervenants de première ligne des groupes communautaires puissent acquérir certaines connaissances pertinentes et assurer une meilleure solidarité entre eux sur le plan régional. Nous en présentons ici la démarche et les méthodes ainsi que quelques résultats fragmentaires. 


\section{Appauvrissement, aide alimentaire et organismes communautaires De la compréhension à l'action}

Élaine CÔTÉ Coordonnatrice des programmes à Moisson Québec

Sonia RACINE Professionnelle de recherche pour Moisson Québec et le Centre de santé publique de Québec Joël ROUFFIGNAT Professeur, Département de géographie et Centre de recherche en aménagement et développement (CRAD)

\section{UNE QUESTION DE BESOINS}

\section{Un contexte...}

La croissance des demandes d'aide alimentaire dans I'agglomération de Québec est à l'origine de ce projet. En effet, depuis sa création en 1988, Moisson Québec, la banque alimentaire régionale, a vu le nombre d'organismes desservis et la quantité de nourriture redistribuée augmenter dramatiquement. Avec des moyens limités, Moisson Québec a pu répondre à ces appels sans cesse croissants. Cette augmentation quasi exponentielle des demandes de secours 
alimentaire a suscité de nombreuses interrogations sur leur origine et leur nature.

Les contraintes économiques récentes ont provoqué une montée du chômage et de l'aide sociale, l'endettement de l'État ainsi que la remise en question de programmes sociaux et médicaux que d'aucuns croyaient établis pour longtemps. De nouvelles formes de gestion de la main-d'œuvre sont apparues. Elles ont provoqué une érosion des conditions traditionnelles du travail: l'emploi permanent et à plein temps avec un salaire régulier et des avantages sociaux a été remis en question. L'organisation flexible de la main-d'œuvre a vu se développer le travail temporaire, contractuel, saisonnier, à temps réduit ou partagé, la sous-traitance, l'emploi individuel (self-employment), la retraite progressive, etc. Cette fragmentation des tâches et des temps du travail se traduit aussi dans des cycles de vie segmentés au cours desquels alternent emplois à temps plein et temporaire, emplois multiples et travail au noir, chômage, aide sociale et programmes "d'employabilité ». Pour reprendre l'image formulée par le sociologue Paul Grell (Grell, 1995), un gradient s'est établi entre la cité sainte et les banlieues du travail, entre l'intégration totale à la société de production-consommation et les périphéries de la précarité, de la marginalité et de l'exclusion. Qu'un tel gradient ait toujours existé, cela est évident. Qu'il ait été encore plus marqué lors de crises antérieures, $c^{\prime}$ est possible. Mais le phénomène est neuf dans son intensité et dans son ampleur dans les pays industrialisés depuis la Seconde Guerre mondiale.

Devant la hausse des cas d'exclusion, les organisations communautaires sont de plus en plus sollicitées afin de prendre en charge certaines activités d'aide sociale assumées autrefois par l'Étatprovidence. Un tel phénomène $n^{\prime}$ est pas totalement négatif puisqu'il pousse les individus à assumer de plus en plus de responsabilités dans leur milieu de vie: la citoyenneté des personnes s'en trouve ainsi renforcée.

Mais ce phénomène suscite aussi des craintes car les organismes communautaires, quelles que soient leurs volontés, leurs énergies et la qualité de leurs actions, demeureront toujours partiels et partiaux dans leurs réponses aux problèmes sociaux. Ils n'ont pas les ressources pour assurer la relève de l'État et pour intervenir avec justesse et justice auprès de tous les groupes vulnérables en tous lieux du territoire.

La montée de l'aide alimentaire est à situer dans ce contexte. Si celle-ci a existé de tout temps dans notre société, elle est par contre devenue plus visible, sinon plus impérative depuis quelques années. Comme ce fut le cas lors des grandes crises économiques passées qui ont vu la distribution de grains et de viandes en Nouvelle-France par les bureaux des pauvres dès 1688 , de produits alimentaires lors de 
la crise des années 1870 , de soupes populaires lors de la crise des années 1930 (Provencher, 1991)...

Aujourd'hui, évolution du système agro-alimentaire oblige, ce sont les banques alimentaires qui sont les actrices principales de I'aide alimentaire. Le mouvement est récent et en pleine expansion. Il est donc tout à fait logique que l'ensemble des intervenants sociaux, économiques et politiques se tournent vers celles-ci dans le débat actuel sur l'appauvrissement de certaines catégories sociales.

La croissance des demandes d'aide alimentaire est liée d'abord à l'augmentation du nombre de ménages vivant sous ce seuil de faible revenu depuis 1990.

Au Québec, de $15 \%$ à $22 \%$ de la population active vit sous le seuil de faible revenu chaque année depuis vingt ans. Ce seuil est calculé par Statistique Canada en fonction des sommes moyennes consacrées aux dépenses essentielles de consommation des ménages canadiens (alimentation, logement et habillement) (Ross et al., 1994).

Selon les enquêtes de Statistique Canada sur les dépenses des ménages canadiens et sur les dépenses alimentaires des familles, les ménages vivant sous ce seuil de faible revenu consacrent environ le cinquième de leur budget disponible à l'alimentation, le poste principal de dépenses demeurant le logement. En 1992, année des dernières enquêtes, une personne vivant sous le seuil de faible revenu dépensait en moyenne 27 \$ par semaine pour se nourrir.

L'insécurité alimentaire ou l'insuffisance de consommation alimentaire est cependant beaucoup plus reliée à la situation de grande pauvreté. Cette notion de grande pauvreté peut être définie comme étant le seuil de revenu minimal au-dessous duquel une personne ne peut plus avoir un mode de vie jugé "acceptable» ou "décent » ou "minimal » dans notre société.

Ce niveau peut être établi à partir du coût minimum qu'une personne doit consacrer à des dépenses de consommation pour obtenir un apport alimentaire adéquat, un logement chauffé et éclairé, un habillement et des soins personnels élémentaires et une vie relationnelle minimale (téléphone et transport en commun). Ces coûts minimums ont été évalués et établis par des groupes, des organismes et des chercheurs (Ross et al.; Dispensaire diététique de Montréal; Fraser Institute). Un tel niveau de consommation correspond au seuil de grande pauvreté : cela signifie que dans des agglomérations de la taille de celles de Québec ou de Montréal, une personne vivant seule dispose d'un revenu annuel inférieur à 6556 \$ par année ou, pour une famille de quatre personnes, inférieur à 14385 \$ (Rouffignat et al., 1996). 
Les personnes disposant de ce niveau de revenu vivent deux types de situation: une situation de grande pauvreté de longue durée, $c^{\prime}$ est-à-dire pendant plusieurs années successives, ou une situation de grande pauvreté de courte durée ou "conjoncturelle» qui peut durer de quelques semaines à quelques mois: étudiants boursiers, personnes en attente $d^{\prime}$ 'une retraite, $d^{\prime}$ une aide sociale, $d^{\prime}$ 'un paiement $\mathrm{d}^{\prime}$ assurance-chômage, etc., ont des revenus momentanément très faibles, sinon inexistants.

Dans la société québécoise, de $5 \%$ à $6 \%$ de la population vit dans de telles situations. La proportion des deux types de pauvreté de longue ou de courte durée a probablement déjà été établie, mais pour notre problématique cela n'a guère d'importance, puisque quelle que soit la durée dans laquelle une personne est en situation de grande pauvreté, il lui faut manger chaque jour: courte et longue durée ont donc peu de signification au regard du dépannage alimentaire.

La croissance des demandes d'aide alimentaire est liée également au processus d'appauvrissement des ménages et des personnes.

Il faut distinguer entre pauvreté et appauvrissement. Ce sont deux notions différentes. La première correspond à un état de fait, à une situation mesurable, même si cette mesure est subjective et peut prêter à discussion. La seconde s'apparente plus à un processus, à une mécanique dans laquelle sont emmaillés les conditions macroéconomiques et les événements sociaux de la vie des individus. Ces événements importants ont des impacts économiques souvent imprévisibles (rupture du couple, perte de l'emploi, naissance d'un enfant, prise de la retraite, accident ou maladie, etc.). La personne qui formule une demande d'aide alimentaire subit ou a subi un appauvrissement, une baisse de son pouvoir d'achat qui la place en état de pauvreté même momentanée. Cette baisse n'est pas seulement le résultat des politiques macro-économiques. Elle s'inscrit concrètement dans les cheminements individuels.

$C^{\prime}$ est dans le cadre de ce double processus d'augmentation de la pauvreté et de multiplication des situations d'appauvrissement que se développent les demandes d'aide auprès des services de dépannage alimentaire.

\section{des acteurs...}

La banque alimentaire régionale, Moisson Québec, a vu le jour en 1986 sous l'impulsion de $M$. André Migneault, et les premières denrées ont été distribuées dans la capitale le 15 novembre 1987. 
Depuis sa fondation, Moisson Québec a vu croître régulièrement le nombre des organismes qu'elle dessert, les quantités qu'elle distribue et les personnes qui reçoivent une aide alimentaire (tableau 1). Depuis, cette croissance s'est stabilisée parce que les dons de nourriture ont plafonné. II n'y a plus assez de denrées pour répondre à toute la demande et Moisson Québec a dû refuser une accréditation à plusieurs organismes et les placer sur une liste d'attente.

\section{TABleau 1}

Nourriture distribuée par Moisson Québec depuis 1988

\begin{tabular}{ccccc}
\hline Année & $\begin{array}{c}\text { Nourriture } \\
\text { recueillie (kg) }\end{array}$ & $\begin{array}{c}\text { Valeur } \\
\text { marchande } \mathbf{( \$ )}\end{array}$ & $\begin{array}{c}\text { Organismes } \\
\text { desservis (Nb) }\end{array}$ & $\begin{array}{c}\text { Fournisseurs } \\
\text { de produits } \\
\text { alimentaires }\end{array}$ \\
\hline 1988 & 500266 & 1885923 & 87 & 35 \\
1989 & 598359 & 2259120 & 112 & 52 \\
1990 & 713353 & 2956578 & 116 & 56 \\
1991 & 1226113 & 4621629 & 138 & 63 \\
1992 & 1762514 & 6504280 & 144 & 70 \\
1993 & 1510370 & 5709199 & 174 & 66 \\
1994 & 1386000 & 5194728 & 173 & 141 \\
1995 & 1485500 & 5567654 & 184 & 79 \\
\hline
\end{tabular}

Au fil des années, la banque alimentaire est devenue un carrefour au sein d'un réseau d'organisations communautaires, publiques et privées. Elle est aussi devenue un lieu de convergence pour les personnes préoccupées de la situation économique, sociale et nutritionnelle de la population.

Elle a acquis ainsi une position stratégique dans la coordination des actions de dépannage menées au sein de ce réseau régional. Des dizaines d'initiatives se font jour chaque année au sein des organismes communautaires. Sans coordination, ces activités deviennent anarchiques et provoquent des effets pervers sur l'ensemble du réseau et des individus qui y œuvrent ou qui bénéficient de leurs interventions. L'esprit de clocher, la duplication des services, la course aux bénéficiaires, l'utilisation abusive des ressources ne sont que quelques-uns des aspects les plus connus.

En même temps que leurs activités prenaient de l'ampleur, les responsables de la banque alimentaire ont vu ces problèmes se développer. En octobre 1991, André Migneault a réuni divers intervenants 
de la région de Québec autour d'une table (devenue depuis lors la Table d'interaction sur la faim de Québec) pour y débattre de cette augmentation des besoins d'aide alimentaire dans la région. Venant des milieux de la santé, de l'information, des églises, de l'éducation, des administrations municipales ou provinciales, des services sociaux, de groupes sociaux divers ou agissant à titre privé, ces personnes se sont donné pour objectif $d^{\prime}$ agir sur ce qu'il est convenu d'appeler la faim dans notre société, c'est-à-dire des problèmes de sous-alimentation. Nous employons ce terme de sous-alimentation plutôt que celui de malnutrition, car celle-ci peut être due à une surconsommation de nourriture (problèmes d'obésité) ou à une consommation de denrées inadéquates (consommation trop abondante de corps gras, de sel, de junk-food, etc.).

\section{des questionnements...}

Jusqu'en 1992, les responsables de Moisson Québec et de la Table $d^{\prime}$ interaction sur la faim de Québec se sont contentés d'assurer le développement des services et activités d'aide alimentaire au sein des divers organismes régionaux en place ou en émergence (tableau 2). Mais devant la croissance quasi exponentielle des besoins et la dégradation de la situation socio-économique, certaines questions ont dû être soulevées:

1. Parvenu à un tel niveau de développement, le réseau de dépannage alimentaire apparaît plus comme une béquille à la réduction des services sociaux que comme une solution temporaire à des problèmes d'insécurité alimentaire.

2. La multiplication des services de dépannage alimentaire, bien qu'elle provienne $d^{\prime} u n$ bénévolat fort généreux, provoque une dispersion et un chevauchement des activités et fait peser une grave surcharge sur les ressources communautaires disponibles.

3. Les organismes communautaires peuvent difficilement se doter de moyens de vérification des véritables besoins des usagers et de normes de distribution. Le fait de fournir une aide alimentaire à toute personne qui se dit dans le besoin ne permet pas d'orienter les efforts en direction des groupes les plus vulnérables sur le plan alimentaire, nutritionnel et de la santé: il leur faut pouvoir évaluer les besoins réels à la fois sur le plan économique et sur le plan nutritionnel.

4. Le dépannage alimentaire a une grande capacité d'attraction pour les gens en situation précaire. $C^{\prime}$ est souvent la première 
forme d'aide sollicitée par une personne en détresse et il existe un fort potentiel pour associer à celle-ci d'autres types d'intervention à long terme permettant de résoudre des problèmes personnels d'ordre psychologique, social, économique et de santé. Bien organisée et bien insérée dans un ensemble de services, I'aide alimentaire fournit ainsi un excellent levier $d^{\prime}$ intervention de première ligne auprès des personnes et des groupes vulnérables qui y ont recours.

Ce quadruple questionnement sur l'aide alimentaire, sur sa finalité, son organisation, ses limites et ses relations avec les autres types d'intervention est au centre de la réflexion collective amorcée à Moisson Québec et à la Table d'interaction sur la faim de Québec. $C^{\prime}$ est ce questionnement qui a conduit à l'élaboration d'une recherche conscientisante sur I'aide alimentaire dans I'agglomération de Québec.

\section{TABleau 2}

Évolution des quantités de nourriture distribuées par Moisson Québec selon le type de clientèle desservie par les organismes, 1988-1994

\begin{tabular}{|c|c|c|c|c|c|c|c|c|}
\hline & 1988 & 1989 & 1990 & 1991 & 1992 & 1993 & 1994 & 1995 \\
\hline Clientèle variée & 133241 & 141951 & 192656 & 447085 & 705625 & 707878 & 634317 & 680349 \\
\hline Familles à faible revenu & 38060 & 58806 & 81964 & 142384 & 149106 & 162606 & 182.279 & 191861 \\
\hline Familles monoparentales & 5637 & 7609 & 20190 & 34280 & 42899 & 45410 & 35959 & 40793 \\
\hline Femmes en difficulté & 9034 & 8979 & 10756 & 31300 & 32019 & 40097 & 36720 & 34528 \\
\hline Jeunes et enfants & 10269 & 11831 & 17964 & 22121 & 50906 & 70415 & 75868 & 106441 \\
\hline Santé mentale & 10424 & 14374 & 13024 & 21402 & 31428 & 55264 & 57694 & 41082 \\
\hline Toxicomanes & 54121 & 67451 & 61211 & 91200 & 148403 & 131784 & 128750 & 119575 \\
\hline $\begin{array}{l}\text { Autres (immigrants, } \\
\text { sourds, etc.) }\end{array}$ & 91911 & 57897 & 66273 & 134893 & 207473 & 210151 & 202972 & 250192 \\
\hline $\begin{array}{l}\text { Organismes divers } \\
\text { (écoles, CLSC, etc.) }\end{array}$ & 10869 & 25441 & 23492 & 26076 & 20702 & 19012 & 16469 & 6588 \\
\hline Total & 365554 & 396328 & 489520 & 952732 & 1390553 & 1444610 & 1373022 & 1473404 \\
\hline
\end{tabular}

\section{...un projet}

La réalisation d'une recherche conscientisante a été retenue pour que la banque alimentaire régionale dispose de connaissances sur la dynamique de l'aide alimentaire tant sur le plan des faits que sur celui des processus et des acteurs. Ces connaissances doivent lui permettre de mieux effectuer son travail de coordination à long terme. 
Les responsables et les bénévoles des organismes communautaires agissent perpétuellement en situation d'urgence. Ils n'ont ni le temps ni les ressources ni les moyens de prendre du recul par rapport à leurs actions pour en analyser la nature, la pertinence et les retombées. La situation étant nouvelle et changeante, il y avait là un besoin de connaissances sur les divers aspects du phénomène, car la recherche sur la sous-alimentation et sur l'aide alimentaire dans notre société est beaucoup plus limitée que celle sur la pauvreté.

Les conséquences de la sous-alimentation sur la santé physique et mentale et sur la qualité de vie des citoyens des pays dits développés sont difficiles à cerner. Comme le soulignaient dans leur rapport les responsables de la Physician Task Force on Hunger in America de la Harvard School of Public Health (1985):

The data we have discussed on poverty, malnutrition and ill-health is not always easy to assess. There are gaps we would like to see filled and relationships that we can only guess at rather than prove. Lack of baseline data for many problems makes it hard to pinpoint trends over time (Hunger in America, 1985 : 129).

Pour les besoins régionaux, trois grands axes de recherche ont été retenus lorsque les membres de la Table d'interaction sur la faim ont décidé de conduire une recherche conscientisante dans I'agglomération de Québec:

1. Développer et améliorer les actions d'aide alimentaire de Moisson Québec par une meilleure connaissance de son milieu $d^{\prime}$ intervention et par une conscientisation accrue des personnes qui s'impliquent dans ce milieu. Cet objectif exige une meilleure connaissance des besoins du réseau des organismes communautaires offrant des services d'aide alimentaire et une meilleure connaissance des usagers qu'ils desservent. Moisson Québec espère ainsi optimiser ses actions en fonction de la rareté de ses ressources.

2. Avoir une meilleure connaissance des relations qui existent entre l'appauvrissement de certains groupes sociaux et leurs besoins en aide alimentaire. Cet objectif vise à permettre de planifier le travail d'approvisionnement de Moisson Québec avec plus d'efficacité vers les groupes les plus vulnérables de la société.

3. Se doter de moyens efficaces d'intervention au niveau régional auprès des groupes et personnes desservis, mais aussi auprès des instances administratives (CLSC, Régie régionale de la santé et des services sociaux) et gouvernementales (municipalités, 
ministères provinciaux et fédéraux). Moisson Québec et la Table d'interaction sur la faim de Québec veulent ainsi jouer le rôle d'organismes responsables dans les interventions et débats entourant la question de la sous-nutrition et de l'insécurité alimentaire dans notre société et notre région.

Afin de parvenir à ces objectifs généraux, six objectifs spécifiques de recherche ont été établis.

1. Évaluer le réseau actuel des organismes offrant de l'aide alimentaire sur le territoire de l'agglomération urbaine de Québec.

2. Dresser un portrait des usagers de l'aide alimentaire.

3. Analyser l'émergence de la demande d'aide alimentaire parmi les groupes les plus vulnérables de la population régionale.

4. Évaluer les relations entre la demande d'aide alimentaire et les autres besoins personnels et matériels.

5. Conscientiser et informer les responsables communautaires par une meilleure connaissance des besoins et des services d'aide et de dépannage alimentaire.

6. Favoriser le développement de mécanismes de concertation et de collaboration entre les organismes offrant des services $d^{\prime}$ aide et de dépannage alimentaire.

\section{UNE QUESTION DE MÉTHODE}

\section{Un type de recherche...}

Parce que la problématique émane des besoins du milieu, la méthodologie retenue pour ce projet est celle de la recherche conscientisante. Ce type de recherche permet de "recueillir, articuler les données, briser les tabous (interroger "ce qui va de soi"), favoriser la réflexion systématique, s'attacher à découvrir les opinions, les systèmes de référence conscients ou inconscients, situer le local dans le global, passer de l'observation à l'analyse et à l'interprétation » (Ampleman, 1987). Elle est un moyen de mobilisation d'un groupe qui permet d'engager des actions transformatrices et d'envisager de nouvelles solutions aux problèmes vécus. Pour $C$. Lesire la recherche conscientisante représente: " [...] I'investigation des conditions sociales d'existence des catégories sociales de milieu populaire, pour que ces catégories prennent conscience des blocages à leur épanouissement et se mobilisent contre ces blocages" (Mayer et Ouellet, 1991). 
Le but de la recherche conscientisante est de susciter une plus grande prise de conscience de la situation et une action transformatrice qui devrait en découler. Ce type de recherche part de l'action des groupes et elle doit éclairer cette action. Le groupe visé dans ce projet est composé des divers intervenants travaillant au dépannage alimentaire dans la région de Québec. Nous avons signalé que 184 organismes effectuent sous une forme ou sous une autre un tel travail. Certains d'entre eux ont des structures régionales (Conférences SaintVincent-de-Paul, Maisons de Job), d'autres sont purement sectoriels (MIELS-Québec), territoriaux (Fondation Partage chrétien de Loretteville) ou ponctuels (Café Évangélique). Certains en font le motif principal de leur action (Nutrition Famille, Cuisines collectives Vanier), alors que d'autres $n^{\prime} y$ ont recours que marginalement (La Jonction pour elles, École Boudreau).

La recherche conscientisante nous apparaissait donc comme la méthode de recherche la plus apte pour assurer l'intégration de l'ensemble de ces intervenants à la collecte de l'information et à la diffusion de l'information dans ce réseau d'organismes.

\section{des étapes...}

La recherche comprenait quatre étapes principales. En premier lieu, une enquête exhaustive a été menée dès 1993 auprès des 144 organismes desservis par Moisson Québec. Elle visait à décrire et à évaluer globalement l'ensemble du réseau d'aide alimentaire desservi par la banque. Une telle opération avait déjà été menée à Montréal par Moisson Montréal: connaissance élémentaire de l'organisme (adresse, responsables, charte), de ses ressources financières, matérielles et humaines, de ses modalités de fonctionnement, des services alimentaires qu'il fournit et du fonctionnement de ceux-ci; connaissance aussi des conditions matérielles de la cuisine et du stockage de la nourriture, des besoins de nourriture, des types de services non alimentaires, des types de bénéficiaires visés, des besoins locaux, des moyens de contrôle mis en place, de l'évolution récente des besoins et de la collaboration et de l'intercollaboration avec les organismes et le réseau d'aide sociale du voisinage.

Cette enquête s'est faite par l'intermédiaire du personnel bénévole de Moisson Québec. Le rapport sur ce réseau a été terminé au printemps 1994 . On en a utilisé les informations préliminaires pour planifier la seconde partie du projet qui a consisté en une enquête-sondage de type semi-fermé destinée à tracer le profil des personnes et des ménages qui demandent une aide alimentaire (situation socio-familiale, situation budgétaire [revenus mensuels et 


\section{TABLEAU 3}

\section{Démarche et méthode de la recherche conscientisante}

\section{Objectifs}

1. Évaluer le réseau de dépannage alimentaire de I'agglomération urbaine de Québec.

2. Dresser un portrait des usagers de l'aide alimentaire.

3. Analyser l'émergence de la demande alimentaire.

4. Évaluer les relations entre la demande alimentaire et les autres besoins.

5. Former les responsables des organismes communautaires.

6. Favoriser le développement de mécanismes de concertation et de collaboration entre organismes.

\section{Instruments de recherche}

- Questionnaire fermé

- Enquête-sondage (questionnaire semifermé)

- Récits de vie

- Voir les deux étapes précédentes.

- Rencontres-échanges par secteurs de CLSC (3 rencontres maximum par secteur visé)

- Colloques régionaux sur l'aide alimentaire

\section{Échantillon}

- 144 organismes de la région de Québec offrant un service de dépannage alimentaire

- 285 usagers de divers types de services de dépannage alimentaire (échantillonnage au hasard au sein de 25 organismes) et 53 ménages à faible revenu n'ayant jamais demandé d'aide alimentaire

- 16 usagers de divers types de services de dépannage alimentaire (informateurs clés)

- Voir les deux étapes précédentes.

- Plus de 80 participants représentant une soixantaine d'organismes

- 98 participants représentant 61 organismes au premier colloque et 106 participants représentant 75 organismes au second

dépenses principales], situation alimentaire [évaluée à partir de la Hunger Scale et du Food Security Index du Edmonton Food Policy Council] et la situation sociosanitaire [handicaps, maladies chroniques, fréquentation des services de santé]). Cette enquête-sondage d'une durée maximale de 60 minutes par personne a été menée 
auprès d'un échantillon de 285 ménages (610 personnes) choisis au hasard parmi les utilisateurs des services de dépannage alimentaire dans l'ensemble de la région métropolitaine de Québec ainsi qu'auprès de 53 ménages (117 personnes) n'ayant jamais demandé d'aide alimentaire mais vivant avec un faible revenu.

Pendant que s'effectuait le traitement statistique de ces données, des récits de vie ont été recueillis auprès de 16 bénéficiaires d'un nombre restreint $d^{\prime}$ 'organismes de la région de Québec pour analyser leur processus d'appauvrissement et l'émergence de leur besoin de dépannage alimentaire. Ces entrevues d'une durée moyenne de 90 minutes avaient pour objectif d'analyser quand et comment est formulée la demande d'aide alimentaire, ainsi que d'évaluer la place qu'elle occupe dans la consommation et les stratégies de vie de ces personnes.

Finalement et parallèlement aux récits de vie, la formation des intervenants et l'établissement d'une structure de participation ont été visés par le truchement de deux activités principales: I'organisation de deux colloques régionaux sur l'aide alimentaire ainsi que la mise en place de rencontres-échanges entre organismes effectuant divers types d'aide alimentaire par secteur de CLSC. Cette structure de concertation et de collaboration a été retenue afin de permettre aux organismes directement engagés ou non dans la recherche de venir fournir et recevoir de l'information tant sur le plan conceptuel que factuel.

\section{QUELQUES RÉSULTATS DE LA RECHERCHE}

\section{Les usagers de l'aide alimentaire : situations}

En ce qui a trait au besoin de dépannage alimentaire, ce ne sont pas tant les caractéristiques sociologiques des usagers des banques alimentaires qui importaient que leur degré de marginalisation et d'exclusion dans les domaines économiques, sociaux et de la santé.

Bien sûr, les usagers sont en majorité des personnes vivant des revenus du bien-être social $(74 \%)$, sous le seuil de grande pauvreté $(53 \%)$, avec des revenus personnels disponibles de $20 \$$ par mois par personne lorsque toutes les dépenses essentielles ont été payées (alimentation, logement, habillement et services de téléphone et transport en commun) et que les dettes ont été remboursées. Ils ont une faible scolarisation ( $68 \%$ n'ont pas terminé le secondaire) et peu de formation professionnelle ( $46 \% \mathrm{n}^{\prime}$ en ont aucune). Ils sont souvent isolés socialement (célibataires, divorcés ou séparés, veufs ou veuves) et ne peuvent compter sur un réseau de soutien familial et social très étendu. Plus encore, ces personnes ont eu à faire face au décès (18\%) 
ou à la maladie de personnes proches (29\%) au cours des mois précédant leur demande d'aide.

Leur état de santé montre de nombreuses difficultés physiques: une personne sur cinq souffre de mobilité réduite (handicapés physiques, personnes âgées, troubles visuels). D'autre part, une personne sur dix souffre d'une maladie mentale ou d'une déficience l'empêchant d'avoir une vie active normale. Pour tous les autres types de maladies, le pourcentage des usagers qui en souffrent est nettement supérieur aux résultats obtenus pour l'ensemble de la population québécoise lors des enquêtes de Santé-Québec (tableau 4).

\section{TABleau 4}

Personnes signalant des problèmes de santé (en \%)

\begin{tabular}{lccc}
\hline Problèmes & $\begin{array}{c}\text { Enquête auprès } \\
\text { des usagers } \\
\mathbf{N}=\mathbf{6 1 0}\end{array}$ & $\begin{array}{c}\text { Groupe à } \\
\text { faible revenu } \\
\mathbf{N}=\mathbf{1 1 7}\end{array}$ & $\begin{array}{c}\text { Enquête } \\
\text { Santé-Québec } \\
\mathbf{1 9 8 7}\end{array}$ \\
Physiologiques & 17,2 & 13,7 & 12,5 \\
Respiratoires & 19,2 & 2,6 & 7,2 \\
Cardiovasculaires & 15,7 & 3,4 & 10,4 \\
Digestifs & 18,9 & 5,1 & 7,8 \\
Santé ou déficience & & & 8,8 \\
mentale & 4,8 & 0,0 & 10,1 \\
Anxiété / stress & 34,3 & 19,6 & 46,0 \\
Autres problèmes & 56,8 & 39,4 & \\
\hline
\end{tabular}

Les pratiques d'achats alimentaires montrent que les sommes consacrées à l'épicerie sont très proches des recommandations du Dispensaire diététique de Montréal. Les utilisateurs des services de dépannage alimentaire connaissent cependant une réelle insécurité alimentaire, puisqu'un adulte sur quatre $(29,3 \%)$ et un enfant sur dix $(8,3 \%)$ sont demeurés au moins une journée sans manger au cours du trimestre précédant notre entrevue. Beaucoup diminuent leurs rations ou sautent des repas ( $52 \%$ des adultes et $51 \%$ des enfants). La quasitotalité $(95 \%)$ utilisent le recours au colis de nourriture comme stratégie de survie. La majorité des utilisateurs de services de dépannage jouent sur la panoplie des services en allant alternativement aux soupes populaires, repas communautaires et autres cuisines collectives afin de "compléter » le sac de nourriture collecté à un comptoir. La totalité de cette aide alimentaire correspond environ à $20 \$$ par personne et par mois. 
Parmi les groupes les plus vulnérables, les jeunes adultes (18-35 ans) et les adultes seuls (35-65 ans) connaissent de graves difficultés financières qui les placent en situation très précaire. $D^{\prime}$ après nos résultats, personnes âgées et familles monoparentales sont sans doute les groupes les moins vulnérables et les enfants des familles monoparentales sont en général en situation alimentaire moins précaire que les enfants des autres types de ménages.

Ces quelques bribes de résultats nous indiquent que le processus de marginalisation $n^{\prime}$ est pas seulement économique et social, mais que les personnes qui ont eu des problèmes de santé soit personnellement, soit dans leur famille sont plus touchées que les autres.

La question qui se pose est de savoir comment il se fait que dans une société qui se pique d'avoir l'un des meilleurs systèmes de santé au monde, une partie de la population atteinte de handicaps physiques et mentaux doit recourir aux banques alimentaires pour s'alimenter.

\section{Les usagers de I'aide alimentaire: parcours}

Les récits de vie réalisés avec des personnes utilisatrices de divers services d'aide alimentaire (principalement de colis de nourriture) $n^{\prime}$ ont pas seulement permis de cerner l'émergence de la demande alimentaire, ils ont aussi facilité l'analyse des parcours individuels et familiaux d'appauvrissement, des vécus émotionnels et matériels rattachés à la situation de pauvreté, des potentiels des individus souvent inexploités ainsi que de la place concrète de l'aide alimentaire dans le vécu quotidien de ces personnes.

Concernant l'appauvrissement, nous avons décelé deux grands types de parcours: la pauvreté de "longue durée» et la pauvreté "situationnelle ou récente". Nous les définissons comme étant, dans le premier cas, une pauvreté datant du vécu familial antérieur, ce que I'on nomme souvent, à tort ou à raison, la pauvreté générationnelle (transmise par les générations). Dans le deuxième cas, il s'agit d'une pauvreté nouvelle $\mathrm{n}^{\prime}$ ayant pas été expérimentée auparavant. Notons qu'en ce qui concerne la pauvreté de longue durée, cela ne signifie pas que les personnes n'ont jamais connu d'épisodes de vie en dehors de cette situation, au contraire. La pauvreté se présente comme une situation mouvante dans laquelle s'emmaillent plusieurs éléments de la vie des individus.

Contrairement à ce que l'on pourrait penser, l'appauvrissement de plusieurs personnes ne relève pas uniquement du manque d'emploi ou de la perte d'un emploi. Certes, tous les événements que 
vivent les individus sont solidement arrimés au contexte socioéconomique de notre société. Un individu en perte de ses capacités (santé mentale ou physique), en situation de moins grande disponibilité (familles monoparentales) ou encore en état de fragilisation à la suite d'événements perturbateurs (rupture conjugale, mortalité) se trouve ainsi exclu du marché de l'emploi où la compétition est intense. C'est ce genre de parcours qui a conduit les individus que nous avons rencontrés vers une situation de pauvreté.

Quant à l'émergence de la demande d'aide alimentaire, nos intuitions de recherche nous avaient amenés à penser que celle-ci était probablement déterminée par un moment particulier d'insuffisance de revenus, provoqué par un événement précis, une impasse financière incontrôlable. Bien que cette intuition soit juste, elle $\mathrm{n}^{\prime}$ explique pas totalement le recours à l'aide alimentaire. En effet, l'existence du réseau des services d'aide alimentaire représente un facteur explicatif: sa présence incite les personnes à se tourner vers cette forme d'aide. Leurs besoins sont réels, certes, mais elles ont maintenant intégré cette forme d'aide dans leur stratégie de survie et y recourent plus aisément.

Si l'intensité de la pauvreté peut représenter un élément qui explique le recours à l'aide alimentaire, sa persistance dans le temps en est un autre. L'analyse des récits a dévoilé que le recours à l'aide sociale comme principale source de revenu peut être "suffisante" temporairement, mais à long terme, dès que survient une période plus difficile ou une dépense imprévue (rentrée scolaire, fêtes de Noël, etc.), il est presque inévitable de tomber dans I'endettement sous diverses formes et dans la réduction du poste budgétaire le plus facilement compressible: celui alloué à l'alimentation. La récurrence de ces situations "de crise", générée par le contexte que nous connaissons (allongement de la durée du recours à l'aide sociale et diminution des allocations), explique le recours de plus en plus systématique aux services d'aide alimentaire comme pratique habituelle de dépannage. $C^{\prime}$ est principalement ce processus d'enlisement qui semble être à l'origine de la demande d'aide. La diminution des allocations d'aide sociale, en réduisant le degré de couverture des dépenses essentielles à leur plus simple expression, provoque l'accroissement de ces situations.

\section{Un réseau d'organismes communautaires étendu et diversifié}

Le réseau d'aide alimentaire dans I'agglomération de Québec regroupait 124 organismes différents en 1992, lorsque les sociétés SaintVincent-de-Paul étaient répertoriées individuellement. De ce nombre 
une vingtaine réalisaient plus de la moitié de toute l'aide alimentaire régionale. Ces chiffres $n$ 'ont guère varié depuis cette date.

Ce sont principalement les centres d'hébergement (maisons pour toxicomanes, pour femmes victimes de violence, maisons de transition pour délinquants ou pour ex-psychiatrisés, etc.), les comptoirs alimentaires, les centres de dépannage (offrant essentiellement une aide matérielle) et les centres de jour (offrant plutôt un soutien psychosocial) qui sont les principaux acteurs du dépannage alimentaire (plus de $85 \%$ de l'aide).

\section{TABleau 5}

\section{Classification des organismes par type de services ou d'institutions}

\section{Classification de l'organisme}

Centre d'hébergement

Comptoir alimentaire

Centre de dépannage

Centre de jour

Repas communautaire/

soupe populaire

Cuisine collective

Popote roulante

Total
Nombre

37

26

22

21

9

7

2

124
$\%$

29,84

20,96

17,74

16,94

7,26

5,65

1,61

100,00

La majorité de l'aide alimentaire s'effectue sous la forme de distribution de sacs ou de colis de nourriture. Cette forme est facile à mettre en place et à gérer. $C^{\prime}$ est aussi la plus "aseptisée " des formes $\mathrm{d}^{\prime}$ aide, puisqu'elle réduit au minimum les relations entre donateur et receveur. C'est probablement la moins dynamique des formes d'aide si l'on veut que celle-ci dépasse la simple charité pour déboucher sur des actions d'entraide, de solidarité et de réduction de la précarisation et de la marginalisation de personnes vivant des situations déstabilisantes.

L'hétérogénéité du réseau se reflète également dans la multiplicité des objectifs visés. Certes, près de la moitié des organismes $\mathrm{n}^{\prime}$ ont pour seul objectif que de donner de la nourriture, mais il est cependant très positif de voir que nombre d'entre eux cherchent à rejoindre les personnes isolées ou marginalisées et à amorcer un processus de réinsertion sociale ou de formation individuelle. 


\section{TABLEAU 6}

\section{Services alimentaires offerts par les organismes}

\section{Services alimentaires}

Distribution de colis

Repas en centre d'hébergement

Bons de nourriture

Cuisines collectives

Repas communautaires

Collation

Soupe populaire

Popote roulante

Repas en milieu scolaire

Activités spéciales

Cours de cuisine

Déjeuner

\section{Nombre}

78

41

24

16

15

9

9

8

3

3

2

1
$\%$

62,90

33,06

19,35

12,90

12,10

7,26

7,26

6,45

2,42

2,42

1,61

0,81

Nombre de services alimentaires : 12

Total des organismes : 124 / 100,00\%

\section{Tableau 7}

Nombre d'organismes par type d'objectifs

\section{Objectifs}

Offrir un service de dépannage alimentaire et/ou de repas

Améliorer la qualité de vie, briser l'isolement

Formation personnelle, professionnelle 
La diversité des types d'organismes, des services offerts et des objectifs visés a une contrepartie: la duplication des actions, le chevauchement territorial (plusieurs organismes offrent le même service dans la même municipalité ou dans le même quartier: ainsi, dans la seule basse-ville de Québec, plusieurs dizaines d'organismes sont actifs dans l'aide alimentaire) ou sectoriel (plusieurs organismes " concurrents » desservant des clientèles identiques offrent le même type de service de dépannage alimentaire, l'exemple des groupes de familles monoparentales étant à ce titre tout à fait représentatif).

Contre cet éparpillement des actions et la tension qu'il fait peser sur les ressources collectives en termes de travailleurs permanents ou bénévoles, de ressources en aliments (qui plafonnent depuis quelques années) ou de financement (la "course » aux subventions basée sur le nombre de services offerts par les organismes), une seule action possible: la concertation et la collaboration entre organismes. Cette solution $n^{\prime}$ est pas la plus facile, car il est illusoire d'obtenir la participation de tous les groupes dont un certain nombre ne peuvent ou ne veulent pas s'engager dans un tel processus.

\section{UNE INDISPENSABLE CONCERTATION À RÉALISER}

La concertation qui a été amorcée dans le cadre du projet de recherche et soutenue par la Table d'interaction sur la faim de Québec a engendré à la fois des résultats très concrets dans l'action et des réflexions et questionnements pour la soutenir. Par les rencontreséchanges tenues dans la majorité des secteurs de CLSC de la région de Québec (certains secteurs ont été regroupés afin d'obtenir des structures de participation plus dynamiques) et les deux colloques sur le devenir de l'aide alimentaire, les divers intervenants ont été amenés à remettre en question certaines certitudes, à s'interroger sur leurs actions et leurs résultantes, à partager des informations et des réflexions sur la complexité de l'aide alimentaire et ses difficultés, à échanger des moyens d'action permettant de réellement combattre la pauvreté et tous ses avatars. L'ampleur relativement récente de l'aide alimentaire dans notre région et son accroissement spectaculaire au cours des dernières années suscitent la nécessité $d^{\prime}$ une concertation et d'une collaboration accrue de la part de l'ensemble des organismes qui offrent ce type de service. Ces rencontres et colloques ont donc permis de discuter de l'information amassée et des analyses qui en ont découlé. La démarche amorcée a été plus que bénéfique à la fois pour Moisson Québec, en tant que banque alimentaire de la région, et pour les organismes qui se préoccupent de cette problématique. 
Les liens qui se sont tissés par ses multiples rencontres ont provoqué une vaste remise en question ainsi qu'un redoublement $d^{\prime}$ ardeur à la création d'un réseau cohérent et efficace.

\section{...UNE CONTINUITÉ DANS L'ACTION}

Bien que la recherche conscientisante soit terminée dans les faits, l'action qu'elle a provoquée se poursuit encore aujourd'hui, notamment par la continuité de la concertation par secteur de CLSC. En effet, quelques groupes ont convenu de poursuivre leurs réflexions en continuant de se rencontrer périodiquement. La recherche a donc laissé des traces tangibles dans le réseau d'aide alimentaire de la région de Québec. Deux des regroupements ont même décidé de se rencontrer afin de discuter exclusivement du rapport-synthèse de la recherche, lequel comprend des orientations pour l'action des groupes effectuant de l'aide alimentaire. Soutenues par la Table d'interaction sur la faim de Québec et par Moisson Québec, ces rencontres suscitent beaucoup de réflexions, de remises en question et de controverses, éléments essentiels à l'évolution des services et à leur adaptation aux diverses réalités de la pauvreté telle qu'elle est vécue aujourd'hui.

Ces quelques jalons de résultats de recherche démontrent l'importance de s'attarder à la problématique de la sous-nutrition dans notre société d'abondance. D'autres articles suivront afin de se pencher davantage sur les résultats de cette vaste étude sur l'aide alimentaire et son réseau et sur la nécessité de s'interroger sur cette problématique toujours grandissante. 


\section{Références bibliographiques}

AMPLEMAN, G. (1987). Pratiques de conscientisation 2: logement, alphabétisation, féminisme, aide sociale, syndicalisme, santé, politique. Québec, Saint-Sauveur: Le Collectif, 366 pages.

DisPENSAIRE DIÉTÉTIQUE DE MONTRÉAL (1992). Budget de subsistance et budget de confort minimum. Montréal: Dispensaire diététique de Montréal, annuel, 45 pages.

GRELL, P. (1995). "Centre et banlieue du travail», dans J.-L. Klein, Contre l'exclusion: repenser l'économie: Actes du $13^{e}$ colloque de l'Association d'économie politique. Sainte-Foy: Presses de I'Université du Québec, 319 pages.

MAYER, R. et F. OUELLET (1991). Méthodologie de recherche pour les intervenants sociaux. Boucherville: Gaëtan Morin Éditeur, 537 pages.

ODERKIRK, J. (1992). "Les banques alimentaires", dans Tendances sociales canadiennes, printemps: 6-14.

Olson, K. (1992). Food Security In Edmonton. Organizing for Action. Edmonton: Edmonton Food Policy Council, 64 pages.

PhySiCIAN TASK FORCE ON HUNGER IN AMERICA (1985). Hunger in America: the Growing Epidemic. Middletown, Connecticut: Wesleyan University Press, 231 pages.

ProvenCHER, J. (1991). Chronologie du Québec. Montréal : Boréal, 217 pages.

RICHES, G. (1986). Food Banks and the Welfare Crisis. Ottawa: Conseil canadien de développement social, 171 pages.

Ross, D.P., E.R. SHILlington et C. LOCKHEAD (1994). Données de base sur la pauvreté au Canada. Ottawa : Conseil canadien de développement social, 168 pages.

ROUfFIGNAT, J., E. CÔTÉ et S. RACINE (1996). Appauvrissement, aide alimentaire et organismes communautaires. De la compréhension à l'action. Rapport de recherche. Québec: Table d'interaction sur la faim de Québec, 284 pages.

SARLO, C.A. (1992). Poverty in Canada. Vancouver: Fraser Institute, 58 pages.

STATISTIQUE CANADA (1993). Enquête sur les dépenses alimentaires des familles en 1992 : fichier de microdonnées à grande diffusion. Ottawa : Statistique Canada, Section des enquêtes sur les dépenses des familles, Division des enquêtes-ménages, disque d'ordinateur haute densité.

STATISTIQUE CANADA (1994). Enquête sur les dépenses des familles en 1992 : fichier de microdonnées à grande diffusion. Ottawa : Statistique Canada, Section des enquêtes sur les dépenses des familles, Division des enquêtes-ménages, disque d'ordinateur haute densité. 